\section{Frecuencia de riesgo de desnutrición según la Escala de Tamizado para Desnutrición (MST) en un servicio de Medicina Interna}

\author{
VIVIANA CRUZ ${ }^{1, \mathrm{a}}$, LAURA BERNAL ${ }^{2}$, \\ GIANCARLO BUITRAGO ${ }^{1}$, ÁLVARO J. RUIZ ${ }^{1,2}$
}

\section{Screening for malnutrition among hospitalized patients in a Colombian University Hospital}

\begin{abstract}
Background: On admission, 30 to 50\% of hospitalized patients have some degree of malnutrition, which is associated with longer length of stay, higher rates of complications, mortality and greater costs. Aim: To determine the frequency of screening for risk of malnutrition in medical records and assess the usefulness of the Malnutrition Screening Tool (MST). Material and Methods: In a cross-sectional study, we searched for malnutrition screening in medical records, and we applied the MST tool to hospitalized patients at the Internal Medicine Wards of San Ignacio University Hospital. Results: Of 295 patients included, none had been screened for malnutrition since hospital admission. Sixty one percent were at nutritional risk, with a higher prevalence among patients with HIV (85.7\%), cancer (77.5\%) and pneumonia. A positive MST result was associated with a 3.2 days increase in length of hospital stay $(p=0.024)$. Conclusions: The prevalence of malnutrition risk in hospitalized patients is high, but its screening is inadequate and it is underdiagnosed. The MST tool is simple, fast, low-cost, and has a good diagnostic performance.
\end{abstract}

(Rev Med Chile 2017; 145: 449-457)

Key words: Length of Stay; Malnutrition; Mass Screening.

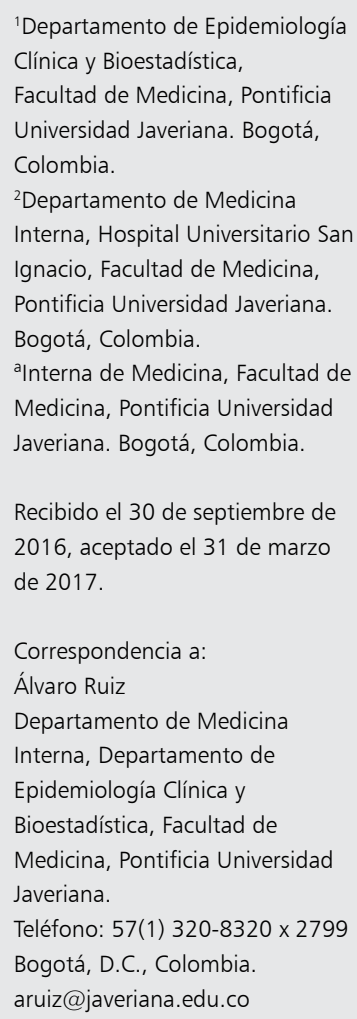

Recibido el 30 de septiembre de 2016, aceptado el 31 de marzo de 2017.

Correspondencia a:

Álvaro Ruiz

Departamento de Medicina Interna, Departamento de Epidemiología Clínica y Bioestadística, Facultad de Medicina, Pontificia Universidad Javeriana.

Teléfono: 57(1) 320-8320 × 2799 Bogotá, D.C., Colombia. aruiz@javeriana.edu.co

$\mathrm{E}$ videncia observacional sugiere que 30 a $50 \%$ de los pacientes presentan algún grado de desnutrición a su ingreso hospitalario ${ }^{1,2}$, con deterioro rápido por suspensión de vía oral, náuseas, efectos medicamentosos o modificaciones dietarias ${ }^{3}$. Cuanto más prolongada es la hospitalización mayor es el riesgo de desnutrición, que puede agregar complicaciones y prolongar la estancia ${ }^{4}$, con aumento en la morbimortalidad y un potencial gran impacto económico en el sistema de salud ${ }^{5,6}$. Lo anterior justifica la evaluación del riesgo de desnutrición en la admisión hospitalaria.

Se han desarrollado y validado múltiples herramientas de tamizado ${ }^{7,8}$, y aunque se reconoce la importancia del tamizado, la difusión es baja y la adherencia irregular y no hay consenso acerca de la mejor herramienta ni sobre cómo elegirla ${ }^{9,10}$. Las más reconocidas son la Valoración Global Subjetiva (SGA, por su nombre en inglés) y la Evaluación Mini Nutricional (MNA) ${ }^{8,11,12}$; no obstante, su aplicación toma un tiempo apreciable ${ }^{13,14}$. Otras son de más fácil aplicación: a destacar, la MNS-SF 
(Mini Nutritional Assesment-short form) es una herramienta validada que consta de 6 ítems, sin embargo, incluye una medida antropométrica (el índice de masa corporal) o en su defecto la circunferencia de la pantorrilla (MNA-SF modificada), para lo cual se requiere de personal entrenado e instrumentos.

La Escala de Tamizado para Desnutrición (MST-Malnutrition Screening Tool) ha demostrado adecuadas características psicométricas en adultos y en ancianos ${ }^{15,16}$. Se desarrolló y validó en pacientes hospitalizados con la SGA como referencia, donde se incluyeron los elementos más importantes de ésta y se excluyeron las medidas antropométricas. Consta de dos sencillas preguntas. Es rápida y puede ser aplicada por cualquier persona, incluidos familiares, sin necesidad de entrenamiento, instrumentos o medidas especiales ${ }^{15,16}$. Se destaca como una herramienta útil para la identificación de pacientes en riesgo de desnutrición ${ }^{10}$, susceptibles de intervenciones nutricionales tempranas ${ }^{3,17,18}$. La MST debe complementarse en los casos positivos con valoración nutricional profesional.

En Colombia no se conoce la utilidad de la escala MST en pacientes hospitalizados ni su frecuencia de uso, por lo tanto, se diseñó este estudio para, por un lado, evaluar la aplicabilidad de esta escala y conocer la frecuencia de su uso espontáneo y, por otro lado, identificar la prevalencia de un resultado positivo y asociar este resultado a desenlaces clínicos relevantes en pacientes hospitalizados en Medicina Interna.

\section{Materiales y Métodos}

Se diseñó un estudio observacional descriptivo de corte transversal que incluyó por conveniencia y secuencialmente a todos los pacientes del servicio de Medicina Interna (urgencias, hospitalización y Unidad de Cuidado Intensivo) del Hospital Universitario San Ignacio (HUSI) entre el 20 al 24 de junio y el 4 al 8 Julio de 2016, que tuvieran diagnóstico de falla cardíaca, enfermedad pulmonar obstructiva crónica, neumonía o síndrome coronario agudo. Se registró si aparecía consignada la MST en la historia clínica; a todos los pacientes se les aplicó la escala. Las respuestas se ingresaron en una planilla electrónica y se registró el tiempo para cada cuestionario. Se tomaron las características demográficas, diagnósticos de hospitalización y comorbilidades. Se registraron también estancia hospitalaria, mortalidad hospitalaria y reingresos a la misma institución hasta el análisis de los datos. Se evaluó la presencia de comorbilidades y se calculó el índice de Charlson (versión abreviada), herramienta diseñada para medir la carga de enfermedad, ponderando las comorbilidades serias, la cual ha mostrado ser predictora de mortalidad ${ }^{19,20}$. Por su gran valor pronóstico, es la más utilizada para clasificar las comorbilidades.

El desenlace principal fue un resultado positivo de la MST (en las primeras $48 \mathrm{~h}$ ), según las recomendaciones de Ferguson (Tabla 1) ${ }^{16}$. Se realizaron análisis descriptivos de la frecuencia de uso de la MST, las características de los pacientes hospitalizados, la frecuencia de resultado positivo según comorbilidades, la estancia hospitalaria y el reingreso al HUSI. Además, se realizaron análisis bivariados para determinar asociaciones entre variables de base al ingreso, estancia hospitalaria, mortalidad durante la hospitalización y reingreso, y el resultado positivo de la MST. Finalmente, con el análisis bivariado se desarrollaron dos modelos multivariados. El primero permitió determinar las características al ingreso asociadas con la presencia del resultado positivo en MST. El segundo buscó la asociación del resultado positivo (ajustado por otras variables) con la probabilidad de muerte durante la hospitalización, y la estancia hospitalaria. Los dos modelos fueron escalonados (stepwise) hacia atrás, para determinar las variables que se incluirían.

Tabla 1. Escala MST

\begin{tabular}{|lc|}
\hline Preguntas & Puntaje \\
\hline $\begin{array}{l}\text { 1. ¿Ha perdido peso de manera involuntaria } \\
\text { recientemente? }\end{array}$ & \\
$\quad$ No & 0 \\
No estoy seguro & 2 \\
Si ha perdido peso ¿cuántos kilogramos? & \\
$1-5 \mathrm{~kg}$ & 1 \\
$6-10 \mathrm{~kg}$ & 2 \\
$11-15 \mathrm{~kg}$ & 3 \\
$>15 \mathrm{~kg}$ & 4 \\
No estoy seguro & 0 \\
2. ¿Ha comido menos de lo normal a causa & \\
de disminución del apetito? & \\
$\quad$ No & 0 \\
Sí & 1 \\
\hline
\end{tabular}


El estudio cumplió las normas éticas internacionales y nacionales; se protegió la identidad de los pacientes. Este estudio es una etapa inicial de un estudio mayor, aprobado por el Comité de Investigación y Ética de la Facultad de Medicina de la Pontificia Universidad Javeriana y del Hospital Universitario San Ignacio.

\section{Resultados}

Se incluyeron 295 pacientes, cuyas características se muestran en la Tabla 2. El 52,2\% eran mujeres y la media para edad fue 58,3 años. No había registro en la historia clínica de evaluación del estado nutricional al ingreso de ninguno de los pacientes, con MST ni con otro instrumento.

Se presentan las cinco causas más frecuentes de hospitalización. Una proporción considerable $(58,6 \%)$ de las hospitalizaciones se debió a infecciones diferentes de las infecciones de vías urinarias (IVU) o neumonía adquirida en la comunidad (NAC), enfermedades autoinmunes, dolor oncológico, enfermedad tromboembólica, pancreatitis o arritmias, entre otras.

El 91\% de los pacientes tenía comorbilidades, siendo la falla cardíaca crónica y cáncer las más frecuentes. El 23\% de los pacientes tuvo alta carga de comorbilidad por índice de Charlson, en especial en mayores de 65 años (Tabla 3). Los pacientes con mayor Charlson fueron aquellos con enfermedad renal crónica. Aproximadamente 50\% de los pacientes con enfermedad pulmonar obstructiva crónica (EPOC), falla cardíaca y diabetes mellitus tuvieron un Charlson correspondiente a alta carga de comorbilidad ( $\geq 3$ ) (Tabla 4$)$.

Todos fueron tamizados por los autores y el promedio de duración de la MST fue 25 segundos. Se encontró que $61,0 \%$ de los pacientes estaban en riesgo de desnutrición, con frecuencias similares en hombres y mujeres (Figura 1). En la

Tabla 2. Características de los pacientes incluidos

\begin{tabular}{|c|c|c|c|}
\hline Características & $\begin{array}{c}\text { Total } \\
\mathrm{n}=295\end{array}$ & $\begin{array}{l}\text { Mujeres } \\
n=141\end{array}$ & $\begin{array}{c}\text { Hombres } \\
n=154\end{array}$ \\
\hline $\begin{array}{l}\text { Edad, años } \\
\quad \text { Promedio (DE) } \\
\text { Rango }\end{array}$ & $\begin{array}{l}58,3(16,8) \\
18-92\end{array}$ & $\begin{array}{l}57,04(17,4) \\
18-92\end{array}$ & $\begin{array}{l}59,5(16,0) \\
18-87\end{array}$ \\
\hline $\begin{array}{l}\text { Causa de hospitalización } \mathbf{n}(\%) \\
\text { Neumonía adquirida en comunidad } \\
\text { Síndrome coronario agudo } \\
\text { Síndrome de falla cardíaca aguda } \\
\text { EPOC exacerbado } \\
\text { Infecciones de vías urinarias } \\
\text { Otras }\end{array}$ & $\begin{array}{cc}29 & (9,8 \%) \\
15 & (5,0 \%) \\
37 & (12,5 \%) \\
19 & (6,4 \%) \\
22 & (7,4 \%) \\
173 & (58,6 \%)\end{array}$ & $\begin{array}{rr}14 & (9,1 \%) \\
5 & (3,5 \%) \\
12 & (8,5 \%) \\
7 & (4,9 \%) \\
9 & (6,3 \%) \\
94 & (66,6 \%)\end{array}$ & $\begin{array}{l}15(9,7 \%) \\
10(6,4 \%) \\
25(16,2 \%) \\
12(7,8 \%) \\
13(8,4 \%) \\
79(51,3 \%)\end{array}$ \\
\hline $\begin{array}{l}\text { Comorbilidades } \mathbf{n}(\mathbf{\%}) \\
\text { Falla cardíaca crónica } \\
\text { Cáncer } \\
\text { Diabetes mellitus } \\
\text { EPOC } \\
\text { Enfermedad renal crónica* } \\
\text { Enfermedades autoinmunes } \\
\text { Infección por VIH } \\
\text { Otras** } \\
\text { Ninguna }\end{array}$ & $\begin{array}{l}82(27,7 \%) \\
80(27,1 \%) \\
54(18,3 \%) \\
53(17,9 \%) \\
37(12,5 \%) \\
19(6,4 \%) \\
14(4,7 \%) \\
30(10,1 \%) \\
26(8,8 \%)\end{array}$ & $\begin{array}{rr}29 & (20,7 \%) \\
41 & (29,0 \%) \\
21 & (15,0 \%) \\
21 & (15,0 \%) \\
13 & (9,2 \%) \\
10 & (7,0 \%) \\
4 & (2,8 \%) \\
18 & (12,7 \%) \\
13 \quad(9,2 \%)\end{array}$ & $\begin{array}{rr}53 & (34,4 \%) \\
39 & (25,3 \%) \\
33 & (23,4 \%) \\
32 & (21,0 \%) \\
24 & (15,6 \%) \\
9 & (5,8 \%) \\
10 & (6,5 \%) \\
12 & (7,8 \%) \\
13 & (8,4 \%)\end{array}$ \\
\hline $\begin{array}{l}\text { Estancia hospitalaria, días*** } \\
\text { Media (DE) } \\
\text { Rango }\end{array}$ & $\begin{array}{c}13(12,0) \\
1-78\end{array}$ & $\begin{array}{c}13,2(13,1) \\
2-78\end{array}$ & $\begin{array}{c}12,7(10,8) \\
1-65\end{array}$ \\
\hline Mortalidad n (\%) & $18(6,0 \%)$ & $8 \quad(5,6 \%)$ & $10 \quad(6,5 \%)$ \\
\hline
\end{tabular}

*Enfermedad renal crónica en prediálisis (TFG $<20$ mg/min/1,72 m²) o diálisis. ${ }^{* *}$ Comorbilidades menores: hipotiroidismo, litiasis, artritis gotosa, entre otras. ${ }^{* * *}$ Estancia al día del análisis de los datos. 18 pacientes aún se encontraban hospitalizados. 
Tabla 3. Comorbilidad medida por Índice de Charlson

\begin{tabular}{|lccc|}
\hline Índice de Charlson (IC)* $\mathbf{n}(\%)$ & $\begin{array}{c}\text { Total } \\
\mathbf{n = 2 9 5}\end{array}$ & $\begin{array}{c}\text { Mujeres } \\
\mathbf{n = 1 4 1}\end{array}$ & $\begin{array}{c}\text { Hombres } \\
\mathbf{n}=\mathbf{1 5 4}\end{array}$ \\
\hline Sin comorbilidad (IC $=0)$ & $76(25,7 \%)$ & $39(27,6 \%)$ & $37(24,0 \%)$ \\
Baja comorbilidad (IC 1-2) & $150(50,8 \%)$ & $77(54,6 \%)$ & $73(47,5 \%)$ \\
Alta comorbilidad (IC $\geq 3)$ & $69(23,4 \%)$ & $25(17,8 \%)$ & $44(28,5 \%)$ \\
\hline
\end{tabular}

*Versión abreviada19,20.

Tabla 4. Frecuencia de alta comorbilidad por subgrupos

\begin{tabular}{|lccc|}
\hline & Total & Mujeres & Hombres \\
\hline Mayores de 65 años & $43 / 110(39,0 \%)$ & $18 / 48(37,5 \%)$ & $26 / 62(41,9 \%)$ \\
\hline Menores de 65 años & $25 / 185(13,5 \%)$ & $7 / 96(7,2 \%)$ & $18 / 89(20,2 \%)$ \\
\hline EPOC & $27 / 53(50,9 \%)$ & $9 / 21(42,8 \%)$ & $18 / 32(56,2 \%)$ \\
\hline Falla cardíaca crónica & $45 / 82(54,9 \%)$ & $14 / 29(48,3 \%)$ & $31 / 53(58,5 \%)$ \\
\hline Enfermedad renal crónica & $27 / 37(72,9 \%)$ & $9 / 13(69,2 \%)$ & $18 / 24(75,0 \%)$ \\
Diabetes mellitus & $26 / 54(48,1 \%)$ & $6 / 21(28,5 \%)$ & $20 / 33(60,6 \%)$ \\
\hline
\end{tabular}

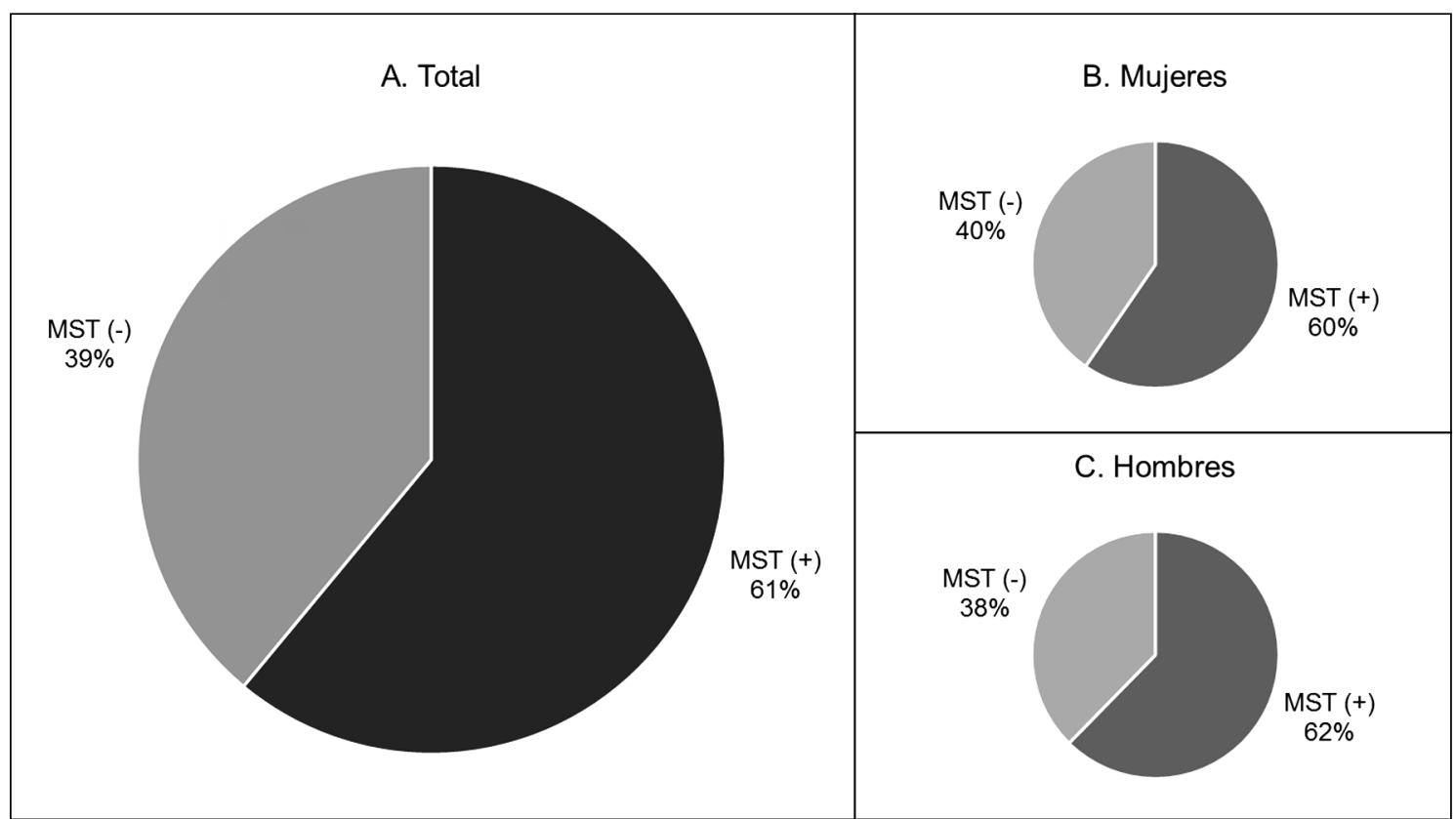

Figura 1. Distribución de resultados de la MST. Distribución de positivos de la MST. A: Todos los pacientes incluidos ( $\mathrm{n}=295)$; B: Mujeres $(n=141)$; C: Hombres $(n=154)$. 
Tabla 5. Frecuencia de resultado positivo de la MST por subgrupos

\begin{tabular}{|c|c|c|c|}
\hline Características & Total & Mujeres & Hombres \\
\hline \multicolumn{4}{|l|}{ Según comorbilidades (n \%) } \\
\hline Población de estudio & $180 / 295(61,0 \%)$ & $84 / 141(59,5 \%)$ & $96 / 154(62,3 \%)$ \\
\hline $\mathrm{VIH}$ & $12 / 14(85,7 \%)$ & $3 / 4(75,0 \%)$ & $9 / 10(90,0 \%)$ \\
\hline Cáncer & $62 / 80(77,5 \%)$ & $33 / 41(80,4 \%)$ & $29 / 39(74,3 \%)$ \\
\hline EPOC & $31 / 53(58,4 \%)$ & $13 / 21(62,0 \%)$ & $18 / 32(56,2 \%)$ \\
\hline Falla cardíaca crónica & $47 / 82(57,3 \%)$ & $17 / 29(58,6 \%)$ & $30 / 53(56,6 \%)$ \\
\hline Diabetes mellitus & $30 / 54(55,5 \%)$ & $12 / 21(57,1 \%)$ & $18 / 33(54,5 \%)$ \\
\hline Enfermedad renal crónica & $16 / 37(43,2 \%)$ & $7 / 13(53,8 \%)$ & $9 / 24(37,5 \%)$ \\
\hline Enfermedades autoinmunes & $9 / 19(47,3 \%)$ & $4 / 10(40,0 \%)$ & $5 / 9(55,5 \%)$ \\
\hline \multicolumn{4}{|l|}{ Según causa de hospitalización (n \%) } \\
\hline Neumonía adquirida en la comunidad & $21 / 29(72,4 \%)$ & $10 / 14(71,4 \%)$ & $11 / 15(73,3 \%)$ \\
\hline Síndrome coronario agudo & $8 / 15(53,3 \%)$ & $1 / 5(20,0 \%)$ & $7 / 10(70,0 \%)$ \\
\hline Falla cardíaca aguda & $21 / 37(56,7 \%)$ & $7 / 12(58,3 \%)$ & $14 / 25(56,0 \%)$ \\
\hline EPOC exacerbado & $11 / 19(57,9 \%)$ & $4 / 7(57,1 \%)$ & $7 / 12(58,3 \%)$ \\
\hline Infección de vías urinarias & $12 / 22(54,5 \%)$ & $5 / 9(55,5 \%)$ & $7 / 13(53,8 \%)$ \\
\hline \multicolumn{4}{|l|}{ Según Índice de Charlson (IC) (n \%) } \\
\hline Sin comorbilidad IC $=0$ & $46 / 76(60,5 \%)$ & $19 / 39(48,7 \%)$ & $26 / 37(70,2 \%)$ \\
\hline Baja comorbilidad IC 1-2 & $91 / 150(60,6 \%)$ & $47 / 77(61,0 \%)$ & $44 / 73(60,2 \%)$ \\
\hline Alta comorbilidad IC $\geq 3$ & $43 / 60(71,6 \%)$ & $17 / 25(68,0 \%)$ & $26 / 35(74,2 \%)$ \\
\hline
\end{tabular}

Tabla 5 se detalla la prevalencia de MST positiva según comorbilidades, esta fue superior para pacientes con VIH $(85,7 \%)$ y cáncer $(77,5 \%)$. Por causas de hospitalización, la prevalencia más elevada fue en neumonía $(72,4 \%)$.

El promedio de estancia fue 13 días, con 11,5 para los pacientes con MST negativo y 13,9 para los pacientes con MST positivo; fue también mayor en aquellos con alta carga de comorbilidad (14,9 días) y aún más en aquellos con alta carga de comorbilidad y MST positivo (15,5 días). El $67,4 \%$ tuvo una estancia superior a 7 días y $8,4 \%$ mayor de 30 . La proporción de pacientes en riesgo de desnutrición varió significativamente según la duración de la hospitalización, con $59,1 \%$ en los de estancia inferior a 7 días, $62,3 \%$ en estancias superiores a 7 días y $72,0 \%$ en las superiores a 30 días. El 15,9\% de los pacientes tuvo algún reingreso en los siguientes 30 días, de los cuales 70,2\% tenían un resultado positivo de MST. Fallecieron 18 pacientes durante la hospitalización, 55\% con alta comorbilidad y $83,3 \%$ con un resultado positivo de MST. El $61,1 \%$ de los pacientes fallecidos tenía cáncer;
5 de los 7 fallecidos sin cáncer estaban en riesgo de desnutrición.

La primera aproximación del análisis multivariado permitió determinar las características asociadas con resultado positivo de la MST. La Tabla 6 presenta los resultados de un modelo multivariado de regresión logística que muestra que edad, tener VIH o cáncer aumentan la probabilidad del resultado positivo de la MST $(\mathrm{p}<0,05)$.

La segunda aproximación de los modelos buscó la asociación del resultado positivo de MST con dos desenlaces clínicos de interés: mortalidad durante la hospitalización y tiempo de estancia. En relación con el primer desenlace, a pesar de que la aproximación escalonada (con probabilidad de entrada de 0,2) incluyó el MST en el modelo final, el resultado positivo de MST no mostró asociación con la probabilidad de morir durante la estancia hospitalaria ( $\mathrm{p}=0,198)$ (Tabla 7). Finalmente, la Tabla 8 presenta los resultados del modelo multivariado lineal que relaciona el resultado de la MST con la estancia hospitalaria. El resultado positivo de la MST aumenta en 3,2 días la estancia hospitalaria, ajustada por otras variables $(\mathrm{p}=0,024)$. 
Tabla 6. Modelo multivariado para determinar la asociación entre características al ingreso y el resultado positivo de la MST

\begin{tabular}{|lccc|}
\hline MST (+) & Odds Ratio & IC 95\% & Valor $\mathbf{p}$ \\
\hline Edad & 1,023 & {$[1,008-1,039]$} & 0,003 \\
VIH (comorbilidad) & 5,595 & {$[1,158-27,017]$} & 0,032 \\
Cáncer (comorbilidad) & 2,880 & {$[1,561-5,314]$} & 0,001 \\
\hline ERC & 0,580 & {$[0,278-1,213]$} & 0,149 \\
NAC & 2,099 & {$[0,847-5,198]$} & 0,109 \\
Constante & 0,293 & {$[0,112-0,761]$} & 0,012 \\
\hline
\end{tabular}

Tabla 7. Modelo multivariado para determinar la asociación entre un resultado positivo de la MST y la mortalidad

\begin{tabular}{|lccc|}
\hline Mortalidad & Odds Ratio & IC 95\% & Valor $\mathbf{~}$ \\
\hline MST (+) & 2,403 & {$[0,630-9,165]$} & 0,198 \\
\hline Días de hospitalización & 1,038 & {$[1,009-1,067]$} & 0,013 \\
\hline Índice de Charlson (+) & 3,765 & {$[1,351-10,488]$} & 0,010 \\
Cáncer (comorbilidad) & 3,259 & {$[1,135-9,354]$} & 0,045 \\
\hline Constante & 0,006 & {$[0,001-0,029]$} & 0,000 \\
\hline
\end{tabular}

Tabla 8. Modelo multivariado para determinar la asociación entre un resultado positivo de la MST y los días de estancia hospitalaria

\begin{tabular}{|lccc|}
\hline Mortalidad & Coeficiente & IC 95\% & Valor $\mathbf{p}$ \\
\hline MST $(+)$ & 3,203 & {$[0,416-5,991]$} & 0,024 \\
\hline Edad & $-0,156$ & {$[-0,241--0,070]$} & 0,000 \\
Índice de Charlson $(+)$ & 4,577 & {$[1,212-7,943]$} & 0,008 \\
Constante & 1,906 & {$[1,403-2,409]$} & 0,000 \\
\hline
\end{tabular}

\section{Discusión}

Múltiples estudios han establecido que el riesgo de desnutrición en pacientes hospitalizados se asocia con peores desenlaces. Aunque es difícil controlar otros factores como severidad de la enfermedad, las publicaciones sugieren que la desnutrición es determinante de desenlaces desfavorables ${ }^{15,18,21}$.

Un estudio de 3.122 pacientes en 56 hospitales de Australia y Nueva Zelandia mostró que los pacientes con desnutrición (detectada por SGA) o con disminución de la ingesta tenían mayor estancia, más reingresos y mayor mortalidad ${ }^{22}$. En
Colombia se encontraron resultados similares en 2015: se aplicó la escala MST a 70 pacientes hospitalizados y se encontró asociación entre el riesgo de desnutrición, estancia hospitalaria y reingresos ${ }^{23}$. Los resultados de nuestro estudio son consistentes con estos resultados.

A pesar de la información que asocia riesgo de desnutrición con mayor morbimortalidad y con mayores costos, el tamizado es limitado. En nuestro estudio se destaca como hallazgo principal la ausencia completa de tamizado nutricional, a pesar de las recomendaciones. El tiempo que podría tomar el tamizado es una de las razones más frecuentes, especialmente en servicios con 
alta demanda. Nuestro estudio evidenció que la duración promedio de aplicación de la MST fue 25 segundos, lo que refuta ese argumento. La evaluación nutricional se hace con frecuencia sólo si hay riesgos evidentes, aunque son precisamente los pacientes en quienes aún no es evidente los que pueden beneficiarse de una intervención temprana. No existe una herramienta ideal de tamizado, pero los atributos deseables para que una escala pueda ser aplicada sistemáticamente es que sea rápida, fácil, reproducible, no invasiva y económica.

Para evaluar las características operativas de las distintas escalas debemos mencionar que no existe un patrón de oro, y se ha definido como patrón de oro operativo la valoración objetiva por un profesional especializado, una valoración nutricional con medidas antropométricas, o las escalas MNA o $\mathrm{SGA}^{8}$. Por las limitaciones de tiempo mencionadas y con la experiencia de que los métodos más completos consumen tiempo que requieren personal entrenado y son más costosas de implementar, se han desarrollado las escalas de tamizado "rápidas y fáciles" como un primer abordaje antes de una evaluación nutricional diagnóstica exhaustiva.

La MST se desarrolló en 1999 con 408 pacientes; se tomaron las preguntas con mayor capacidad de predicción de SGA (pérdida de apetito y pérdida de peso recientes) y se evaluó su concordancia con parámetros nutricionales objetivos. Fue diseñada para ser aplicada por personal no entrenado, en población adulta, tanto para evaluar estado nutricional como para predecir desenlaces clínicos. El estudio reportó menor estancia en los pacientes sin riesgo de desnutrición.

Posteriormente, un estudio comparativo de MST y SNAQ ${ }^{24}$ sugirió que MST tenía mejor validez (sensibilidad de 93\% y especificidad de 93\%), comparado con la valoración por nutricionista y medidas antropométricas como referencia ${ }^{25}$.

Dos estudios posteriores evaluaron la predicción de desenlaces sin que se encontraran resultados positivos; sin embargo, estos estudios se hicieron en ancianos y en pacientes oncológicos y tienen falencias claras ${ }^{26}$. Un estudio evaluó la escala en 130 pacientes oncológicos y solamente mostró alta sensibilidad y especificidad cuando se restringió el análisis a aquellos con alto riesgo nutricional (tumores de cabeza y cuello y gastrointestinales) ${ }^{26}$. Otro estudio en pacientes oncológicos ambulatorios evaluó la concordancia con un estándar aceptable (valoración nutricional completa) y mostró validez aceptable de la escala ${ }^{27}$. En cuanto al estudio en ancianos, se utilizaron métodos discutibles de referencia (prealbúmina y NRS-2002) ${ }^{8}$. El promedio de tiempo gastado en la aplicación de la escala fue menor de un minuto, por personal sin entrenamiento ${ }^{8,10}$.

Nuestro estudio incluyó población adulta heterogénea, con población oncológica bien representada $(27 \%)$, así como otras comorbilidades. Se encontró una prevalencia de riesgo de desnutrición ligeramente superior a la descrita en la literatura (entre $20 \%$ y $50 \%)^{15,22}$. Al excluir la población oncológica o con VIH, la prevalencia fue de $53 \%$, concordante con los estudios publicados, incluso con los colombianos. Es alarmante que, a pesar de la alta prevalencia descrita, la mayoría de los pacientes no son evaluados ni intervenidos desde el punto de vista nutricional ${ }^{2,21}$.

Llama la atención que, entre las causas de hospitalización, la neumonía tuvo el menor Charlson ( 1 de los 29 tuvo un índice alto y 17 no tenían comorbilidad); sin embargo, tuvieron la mayor prevalencia de riesgo de desnutrición y en la regresión logística, esta fue la única causa de hospitalización que se asoció significativamente con un resultado positivo del MST. De las comorbilidades, las asociadas significativamente con riesgo de desnutrición fueron cáncer, VIH y enfermedad renal crónica, lo que permite resaltar la importancia de realizar intervenciones nutricionales en estos grupos.

El riesgo de desnutrición detectado por MST se asoció con mayor estancia, ajustado por otras variables. Más de dos tercios de los pacientes con riesgo de desnutrición y alta carga de comorbilidad tuvieron una estancia superior a 30 días, con un aumento probable en costos directos, indirectos e intangibles ${ }^{19}$. El riesgo de desnutrición se correlacionó también con mortalidad, así como el índice de comorbilidad; aun excluyendo la mortalidad por cáncer, $70 \%$ de los pacientes que fallecieron durante la hospitalización estaban en riesgo de desnutrición.

Este estudio es observacional descriptivo y su principal limitación es la dificultad para ajustar por otras variables, como severidad de la enfermedad de base. Sin embargo, se lograron resultados relevantes. Ya se mencionaron las desventajas de la escala utilizada, pero consideramos que es la que más se ajusta para aplicación rutinaria en todos los ingresos. Debe recalcarse que, si se utiliza el 
MST como herramienta de tamizado, debe estar siempre seguido por una valoración integral en quienes se justifique. Nos proponemos realizar un estudio más amplio y multicéntrico, para obtener prevalencias de riesgo de desnutrición en hospitales universitarios.

\section{Conclusión}

El identificar la prevalencia de desnutrición es un paso fundamental para realizar intervenciones tempranas que puedan modificar los desenlaces. Se justifica la implementación de una herramienta que permita detectar el mayor número de pacientes en riesgo y el seguimiento de la adherencia a esta medida. Se plantea aquí el gran valor de la escala MST, al tratarse de una herramienta sencilla y rápida, que no genera retrasos en la atención médica, y de bajo costo, pero con buen rendimiento diagnóstico y buena capacidad para predecir estancia hospitalaria e incluso mortalidad, así como costos asociados. Su aplicación sistemática en las primeras $48 \mathrm{~h}$ de ingreso al hospital permitiría un diagnóstico mayor, con un impacto significativo en los desenlaces, aunque antes de realizar una recomendación para su uso rutinario, debe realizarse la validación de la versión en español para el contexto colombiano.

\section{Referencias}

1. Merhi VA, de Oliveira MR, Caran AL, Tristão TM, Ambo RM, Tanner MA, et al. [Hospitalization period and nutritional status in hospitalized patients]. Nutr Hosp 2007; 22 (5): 590-5.

2. Ortiz Saavedra P, Manrique Hurtado H, Solís Villanueva J, Candiotti Herrera M, Ige Afuso M, Torres Ruiz C. [Prevalence of malnutrition in the Hospital Medicine Wards]. Rev Soc Peru Med Interna 2007; 20: 16-20.

3. O'Flynn J, Peake H, Hickson M, Foster D, Frost G. The prevalence of malnutrition in hospitals can be reduced: Results from three consecutive cross-sectional studies. Clin Nutr 2005; 24 (6): 1078-88.

4. Ordóñez AM, Schieferdecker MEM, Cestonaro T, Cardoso Neto J, Campos ACL. Nutritional status influences the length of stay and clinical outcomes in patients hospitalized in internal medicine wards. Nutr Hosp 2013; 28 (4): 1313-20.

5. Asiimwe SB, Muzoora C, Wilson LA, Moore CC. Bed- side measures of malnutrition and association with mortality in hospitalized adults. Clin Nutr 2015; 34 (2): 252-6.

6. Amaral TF, Matos LC, Tavares MM, Subtil A, Martins R, Nazaré M, et al. The economic impact of disease-related malnutrition at hospital admission. Clin Nutr 2007; 778-84.

7. Mirmiran P, Hosseinpour-Niazi S, Hamayeli Mehrabani H, Kavian F, Azizi F. Validity and reliability of a nutrition screening tool in hospitalized patients. Nutrition 2011; 27 (6): 647-52.

8. Elia M, Stratton RJ. An analytic appraisal of nutrition screening tools supported by original data with particular reference to age. Nutrition 2012; 28 (5): 477-94.

9. Cooper C, Brierley ER, Burden ST. Improving adherence to a care plan generated from the Malnutrition Universal Screening Tool. Eur J Clin Nutr 2013; 67 (2): 174-9.

10. Van Bokhorst-de van der Schueren MAE, Guaitoli PR, Jansma EP, de Vet HCW. Nutrition screening tools: Does one size fit all? A systematic review of screening tools for the hospital setting. Clin Nutr 2014; 33 (1): 39-58.

11. Lamb CA, Parr J, Lamb EIM, Warren MD. Adult malnutrition screening, prevalence and management in a United Kingdom hospital: cross-sectional study. Br J Nutr 2009; 102 (4): 571-5.

12. Guigoz Y. The Mini Nutritional Assessment (MNA) review of the literature-What does it tell us? J Nutr Health Aging 2006;10 (6): 466-85.

13. Velasco C, García E, Rodríguez V, Frías L, Garriga R, Álvarez J, et al. Comparison of four nutritional screening tools to detect nutritional risk in hospitalized patients: a multicentre study. Eur J Clin Nutr 2011; 65 (2): 269-74.

14. Rice N, Normand C. The cost associated with disease-related malnutrition in Ireland. Public Health Nutr 2012; 15 (10): 1966-72.

15. Lim SL, Ong KCB, Chan YH, Loke WC, Ferguson M, Daniels L. Malnutrition and its impact on cost of hospitalization, length of stay, readmission and 3-year mortality. Clin Nutr 2012; 31 (3): 345-50.

16. Ferguson M, Capra S, Bauer J, Banks M. Development of a valid and reliable malnutrition screening tool for adult acute hospital patients. Nutrition 1999; 15 (6): 458-64.

17. Nursal TZ, Noyan T, Atalay BG, Köz N, Karakayali H. Simple two-part tool for screening of malnutrition. Nutrition 2005; 21 (6): 659-65.

18. Kyle UG, Coss-Bu JA. Nutritional assessment and length of hospital stay. CMAJ 2010; 182 (17): 1831-2. 
19. Deyo RA, Cherkin DC, Ciol MA. Adapting a clinical comorbidity index for use with ICD-9-CM administrative databases. J Clin Epidemiol 1992; 45 (6): 613-9.

20. Gutiérrez-Misis A, Sánchez-Santos M, Otero Á. [Use of a proxy to the Charlson Index to study the short and long-term comorbidity and mortality in the elderly]. Aten Primaria 2012; 44 (3): 153-61.

21. Lim SL, Daniels L. Reply-Malnutrition and its impact on cost of hospitalization, length of stay, readmission and 3-year mortality. Clin Nutr 2013; 32 (3): 489-90.

22. Barker LA, Gout BS, Crowe TC. Hospital malnutrition: Prevalence, identification and impact on patients and the healthcare system. Int J Environ Res Public Health 2011; 8 (2): 514-27.

23. Vesga Varela AL, Gamboa Delgado EM. [Risk of Malnutrition Associated with Poor Food Intake, Prolonged Hospital Stay and Readmission in a High Complexity Hospital in Colombia]. Nutr Hosp 2015;
32 (3): 1308-14.

24. van Venrooij LMW, de Vos R, Borgmeijer-Hoelen AMMJ, Kruizenga HM, Jonkers-Schuitema CF, de Mol BAMJ. Quick-and-easy nutritional screening tools to detect disease-related undernutrition in hospital in- and outpatient settings: A systematic review of sensitivity and specificity. e-SPEN. 2007; 2 (2): 21-37.

25. Neelemaat F, Meijers J, Kruizenga H, Van Ballegooijen $\mathrm{H}$, Van Bokhorst-de van der Schueren M. Comparison of five malnutrition screening tools in one hospital inpatient sample. J Clin Nurs 2011; 20 (15-16): 2144-52.

26. Amaral TF, Antunes A, Cabral S, Alves P, Kent-Smith L. An evaluation of three nutritional screening tools in a Portuguese oncology centre. J Hum Nutr Diet 2008; 21 (6): 575-83.

27. Lancheros-Paez LL, Merchán-Chaverra A, Martínez-Anaya LJ. [Screening nutritional risk in cancer patients]. Rev Fac Med 2014; 62 (S1): 57-64. 